\title{
Functional Test-Sequence Grading at Register-Transfer Level*
}

\author{
Hongxia Fang, Krishnendu Chakrabarty, Abhijit Jas, Srinivas Patil, and Chandra Tirumurti
}

\begin{abstract}
Functional test sequences are often used in manufacturing testing to target defects that are not detected by structural test. Therefore, it is necessary to evaluate the quality of functional test sequences. However, it is very time-consuming to evaluate the quality of functional test sequences by gate-level fault simulation. Therefore, we propose output deviations as a surrogate metric to grade functional test sequences at the register transfer (RT)level without explicit fault simulation. Experimental results for the open-source Biquad filter core and the Scheduler module of the Illinois Verilog Model (IVM) show that the deviations metric is computationally efficient and it correlates well with gatelevel coverage for stuck-at, transition-delay and bridging faults. Results also show that functional test sequences reordered based on output deviations provide steeper gate-level fault coverage ramp-up compared to other ordering methods.
\end{abstract}

Index Terms-Defect, functional test, output deviation, register-transfer level (RT-level), test grading.

\section{INTRODUCTION}

$\mathbf{S}$ TRUCTURAL test offers several advantages over functional test. For example, it allows us to develop test generation and test evaluation (e.g., fault simulation) algorithms based on selected fault models [1]. However, functional test has not yet been completely replaced by structural test. Instead, it is commonly used in industry to target defects that are not detected by structural tests [2]-[4]. An advantage of functional test is that it avoids overtesting since it is performed in normal functional mode. In contrast, structural test is accompanied by some degree of yield loss [5]. Register transfer (RT)-level fault modeling, test generation and test evaluation are therefore of considerable interest [6]-[9].

Given a large pool of functional test sequences (for example, design verification sequences), it is necessary to develop an efficient method to select a subset of sequences for manufacturing testing. Since functional test sequences are much longer than structural tests, it is time-consuming to grade functional test sequences using traditional gate-level fault simulation methods.

The evaluation of functional test sequences is a daunting task if we simply consider just the sheer number of cycles one may have to simulate to evaluate the fault coverage (assuming we know what fault model we are going to grade against). For example, consider a functional test sequence that is equivalent to one second of runtime on a processor with a $3 \mathrm{GHz}$ clock frequency. This means that we have to simulate 3 billion cycles

\footnotetext{
* This work was supported in part by the Semiconductor Research Corporation under Contract no. 1588, and by an equipment grant from Intel Corporation. A preliminary version of this paper was published in Proc. IEEE VLSI Test Symposium, pp. 264-269, 2009.

H. Fang and K. Chakrabarty are with the Department of Electrical and Computer Engineering, Duke University, Durham, NC, 27708 USA (e-mail: $\{$ hf12, krish\}@ee.duke.edu).

A. Jas, S. Patil and C. Tirumurti are with Intel Corporation, Austin, TX, USA (e-mail: \{abhijit.jas, srinivas.patil, chandra.tirumurti\}@intel.com).
}

to evaluate the fault coverage. Even for stuck-at or transition fault models, simulation of the order of a billion cycles on a small fault sample on a reasonably large server farm can take months to complete. Furthermore, for system-level tests that are often created to catch circuit marginality and timing (speed path) errors, if we try to grade these tests on delay fault models, the time taken would be orders of magnitude more than the time needed to grade on transition or stuck-at fault models.

To quickly estimate the quality of functional tests, a highlevel coverage metric for estimating the gate-level coverage of functional tests is proposed in [10]. This metric is based on event monitoring. First, gate-level fault activation and propagation conditions are translated to coverage objects at functional level. Next, a functional simulator is used for monitoring the "hit number" of the coverage objects, and estimating the fault coverage. However, this approach requires considerable time and resources for the extraction of the coverage objects. In particular, experienced engineers and manual techniques are needed to extract the best coverage objects.

In [11], another coverage metric is proposed to estimate the gate-level fault coverage of functional test sequences. This metric is based on logic simulation of the gate-level circuit, and on the set of states that the circuit traverses. It relies on the observation that a test sequence with high fault coverage also traverses a large number of circuit states [12] [13]. The ratio of visited states to the total number of possible states for each subset is used to estimate fault coverage. However, this method does not take the observability of flipflops into consideration. The observability of flip-flops affects the accuracy of the estimated fault coverage, especially when state transitions in the circuit are caused by changes in the contents of sequentially-deep flip-flops. Another drawback of this method is that it is impractical for large designs since it requires gate-level logic simulation.

In this paper, we propose output deviations as a metric at RT-level to grade functional test sequences. The deviation metric at the gate-level has been used in [14] to select effective test patterns from a large repository of $n$-detect test patterns. It has also been used in [15] to select appropriate LFSR seeds for LFSR-reseeding-based test compression. The RTlevel deviation metric has been defined and used in [16]. However, there are two main point of differences between this paper and [16]. Firstly, the problems being targeted in the two papers are different. This paper targets the grading of functional test sequences. The challenge is to identify a computationally tractable and scalable method to rank functional test sequences in terms of their effectiveness for detecting defects in manufacturing testing. In contrast, [16] targeted at a DFT problem: how to select observation point and insert them to increase the quality of a given functional test 
sequence. Second, the basic definitions of output deviations and algorithms used in the two papers are different. In this paper, we use the RT-level output deviations metric to grade the functional test sequences. A single output deviation-based measure is calculated on the basis of each register. In [16], we used a separate definition of RT-level internal deviations and the topological relationship between registers. The deviation is calculated for each bit of a register. Since the focus there was on a single test sequence, more computational effort could be expended.

The remainder of this paper is organized as follows. Section II introduces basic concepts and preliminaries. Section III defines output deviations and describes the procedure used for calculation. We present the experimental results in Section IV. Section V concludes the paper.

\section{Output Deviations at RT-Level: Preliminaries}

In this section, we present basic concepts needed to calculate output deviations at RT-level. Our objective is to use deviations as a surrogate metric for functional test grading.

First, we define the concept of transition count (TC) for a register. Typically, there is dataflow between registers when an instruction is executed and the dataflow affects the values of registers. For any given bit of a register, if the dataflow causes a change from 0 to 1 , we record that there is a $0 \rightarrow 1$ transition. Similarly, if the dataflow causes a change from 1 to 0 , we record that there is a $1 \rightarrow 0$ transition. If the dataflow makes no change to this bit of the register, we record that there is a $0 \rightarrow 0$ transition or a $1 \rightarrow 1$ transition, as the case may be.

After a transition occurs, the value of the bit of a register can be correct or faulty (due to an error). When an instruction is executed, there may be several transitions in the bits of a register. Therefore, an error may be manifested after the instruction is executed. We define the output deviation for instruction $I_{j}$, $\triangle(j)$, as the probability that an error is produced when $I_{j}$ is executed. Section III presents details about the calculation of $\triangle(j)$. The output deviation for an instruction provides a probabilistic measure of the correct operation of instructions at the RT-level.

Similarly, since a functional test sequence is composed of several instructions, we define the output deviation for a functional test sequence to be the probability that an error is observed when this functional test sequence is executed. For example, suppose a functional test sequence, labeled $T_{1}$, is composed of instructions $I_{1}, I_{2}, \ldots, I_{N}$, and let $\triangle(j)$ be the deviation for $I_{j}$, as defined above. The output deviation for $T_{1}, \triangle^{*}\left(T_{1}\right)$, is defined as: $\triangle^{*}\left(T_{1}\right)=\sum_{j=1}^{N} \triangle(j)$. This corresponds to the probability that an error is produced when $T_{1}$ is executed.

Based on these definitions, output deviations can be calculated at RT-level for functional test sequences for a given design. This procedure is described in detail in the next section.

\section{Deviation Calculation at RT-level}

Since our objective is to use deviations as a surrogate metric to grade functional test sequences, we expect test sequences (a)

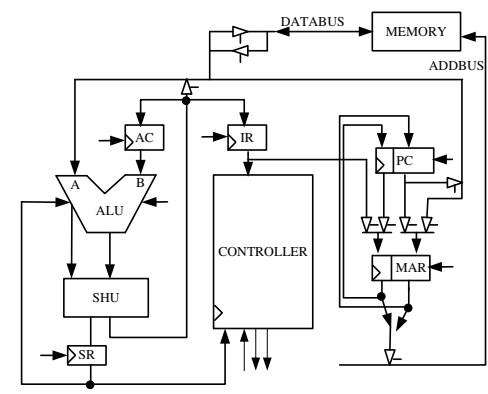

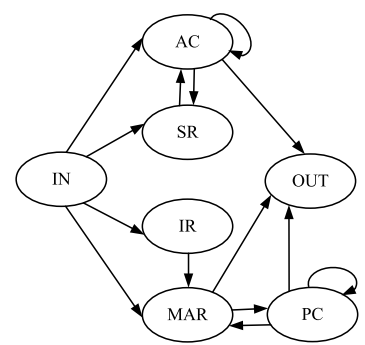

(b)
Fig. 1. (a) Architecture of the Parwan processor; (b) Dataflow graph of the Parwan processor (only the registers are shown).

with higher deviation values to provide higher defect coverage. In order to ensure this, we consider three contributors to output deviations. The first is the transition count (TC) of registers. Higher the TC for a functional test sequence, the more likely is it that this functional test sequence will detect defects. We therefore take TC into consideration while calculating deviations. The second contributor is the observability of a register. The $\mathrm{TC}$ of a register will have little impact on defect detection if its observability is so low that transitions cannot be propagated to primary outputs. The third contributor is the amount of logic connected to a register. In order to have a high correlation between RT-level deviation and gate-level stuck-at fault coverage, we need to consider the relationship between RT-level registers and gate-level components.

In this section, the Parwan processor [17] is used as an example to illustrate the calculation of output deviations.

\section{A. Observability Vector}

We first consider the observability of registers. The output of each register is assigned an observability value. The observability vector for a design at RT-level is composed of the observability values of all its registers. Let us consider the calculation of the observability vector for the Parwan processor [17]. The Parwan is an accumulator-based 8-bit processor with a 12-bit address bus. Its architectural block diagram is shown in Figure 1(a).

From the instruction-set architecture and RT-level description of Parwan, we extract the dataflow diagram to represent all possible functional paths. Figure 1(b) shows the dataflow graph of Parwan. Each node represents a register. The IN and OUT nodes represent memory. A directed edge between registers represents a possible functional path between registers. For example, there is an edge between the AC node and the OUT node. This edge indicates that there exists a possible functional path from register AC to memory.

From the dataflow diagram, we can calculate the observability vector. First, we define the observability value for the OUT node. The primary output OUT has the highest observability since it is directly observable. Using sequential-depth-like measure for observability [1], we define the observability value of OUT to be 0 , written as $O U T \_o b s=0$. For every other register node, we define its observability parameter as 1 plus the minimum of the observability parameters of all its fanout nodes. For example, the fanout nodes of register AC are OUT, 
TABLE I

WEIGHT VECTOR FOR REGISTERS (PARWAN).

\begin{tabular}{|r|r|r|r|r|r|}
\hline & $A C$ & $I R$ & $P C$ & $M A R$ & $S R$ \\
\hline $\begin{array}{r}\text { No. of faults } \\
\text { affecting register }\end{array}$ & 2172 & 338 & 936 & 202 & 2020 \\
\hline Weight value & 1 & 0.1556 & 0.4309 & 0.093 & 0.930 \\
\hline
\end{tabular}

SR, and AC itself. Thus the observability parameter of register $\mathrm{AC}$ is 1 plus the minimal observability parameter among OUT, $\mathrm{SR}, \mathrm{AC}$. That is, the observability parameter of register AC is 1. In the same way, we can obtain the observability parameters for MAR, PC, IR and SR. We define the observability value of a register as the reciprocal of its observability parameter. Finally, we obtain the observability vector for Parwan. It is simply $\left(\frac{1}{A C_{-} o b s} \frac{1}{I R_{-} o b s} \frac{1}{P C_{-} o b s} \frac{1}{M A R_{-} o b s} \frac{1}{S R_{-} o b s}\right)$, i.e., (1 0.5 $110.5)$.

\section{B. Weight Vector}

The weight vector is used to model how much combinational logic a register is connected to. Each register is assigned a weight value, representing the relative sizes of its input cone and fanout cone. The weight vector for a design is composed of the weight values of all its registers. Obviously, if a register has a large input cone and a large fanout cone, it will affect and be affected by many lines and gates. Thus it is expected to contribute more to defect detection. In order to accurately extract this information, we need gate-level information to calculate the weight vector. We only need to report the number of stuck-at faults for each component based on the gate-level netlist. This can be easily implemented without gate-level logic simulation by an automatic test pattern generation (ATPG) tool or a design analysis tool. Here we use Flextest to obtain the number of stuck-at faults for each component in Parwan: there are 248 stuck-at faults in AC, 136 in IR, 936 in PC, 202 in MAR, 96 in SR, 1460 in ALU, and 464 in SHU.

Based on the RT-level description of the design, we can determine the fanin cone and fanout cone of a register. For example, the $\mathrm{AC}$ register is connected to three components: $\mathrm{AC}$, SHU and ALU. Given a set of registers $\left\{R_{i}\right\}, i=1,2, . ., n$, let $f_{i}$ be the total number of stuck-at faults in components connected to register $R_{i}$. Let $f_{\max }=\max \left\{f_{1}, . ., f_{n}\right\}$. We define the weight of register $R_{i}$ as $f_{i} / f_{\max }$ to normalize the size of gate-level logic. Table I shows the numbers of faults affecting registers and weights of registers. We can see that $f_{\max }=2172$ for Parwan processor, which is the number of faults in AC. The weight of IR can be calculated as $338 / 2172$, i.e., 0.1556 . In this way, weights of other registers can also be obtained. Finally, we get the weight vector (1 0.15560 .4309 $0.0930 .930)$.

\section{Threshold value}

The higher the TC is for a functional test sequence, the more likely is it that this functional test sequence will detect defects. However, suppose that the TC is already large enough (greater than a threshold value) after the given functional test sequence is executed for a given number of clock cycles. As the functional test sequence is applied for more clock cycles, TC keeps increasing. However, higher values of TC make no significant contribution to the detection of new defects if they generate only a few new transitions in the registers. The threshold value is used to model what value of TC for a register is useful for defect detection. Each register is assigned a threshold value, representing an upper limit on the TC that is useful for defect detection. While different threshold values can be used for various types of transitions of a register, we assume here without loss of generality that all four types of transitions in a register are assigned identical threshold values. We also assume that all registers are assigned identical threshold values.

The threshold value can be obtained by learning from a few of the given functional test sequences. We only need to run logic simulation at RT-level for these test sequences. Suppose that we have $k$ registers in the design. Our goal is to learn from $m$ functional test sequences $T_{1}, T_{2}, \ldots T_{m}$. The parameter $m$ is a user-defined parameter, and it can be chosen based on how much pre-processing can be carried out in a limited amount of time. In this paper, we use the following steps to obtain the threshold value.

- Run RT-level Verilog simulation for the selected $m$ functional test sequences. Record the new transition counts on all registers for each clock cycle during the execution of the test sequence;

- For each of the $m$ functional test sequences, plot the cumulative new transition count (CNTC). Figure 2 is shown as an example. The $\mathrm{x}$-axis represents the number of clock cycles. The y-axis shows the CNTC, summed over all registers. Our experiments show that this generic plot is representative of benchmark circuits and functional test sequences.

- From the above figure, find the "critical point" on the $x$ axis, after which the CNTC curve flattens out. Formally speaking, the critical point is the $\mathrm{x}$-axis value at which the CNTC reaches $98 \%$ of the maximum CNTC value. For example, from Figure 2, we can see that the critical point is the 130th clock cycle. Denote the critical point for $m$ test sequences as the set $\left\{c p_{1}, \ldots c p_{m}\right\}$;

- Obtain the aggregated TC for all registers up to the critical point. Denote the aggregated TC for $m$ test sequences by the vector $\left(t h_{1} t h_{2} \ldots t h_{m}\right)$. Given $k$ registers in the design, we set the threshold value th to be the average $\left(t h_{1} / k+\ldots t h_{m} / k\right) / m$.

\section{Calculation of Output Deviations}

The output deviations can be calculated for functional test sequences using the TCs, the observability vector, the weight vector and the threshold value. First, we define the countable TCs for registers. Suppose the $0 \rightarrow 0$ TC of register $R_{i}$ for instruction $I_{j}$ is recorded as $t_{i j 00}$. The countable $0 \rightarrow 0 \mathrm{TC}$ of register $R_{i}$ for instruction $I_{j}$ is defined as follows:

$t_{i j 00}^{*}= \begin{cases}0 & \left(\sum_{p=1}^{j-1} t_{i p 00} \geq t h\right) \\ t h-\sum_{p=1}^{j-1} t_{i p 00}\left(\sum_{p=1}^{j-1} t_{i p 00}<t h<\sum_{p=1}^{j} t_{i p 00}\right) & \left(\sum_{p=1}^{j} t_{i p 00} \leq t h\right) \\ t_{i j 00} & \left(\sum_{0}\right)\end{cases}$ 


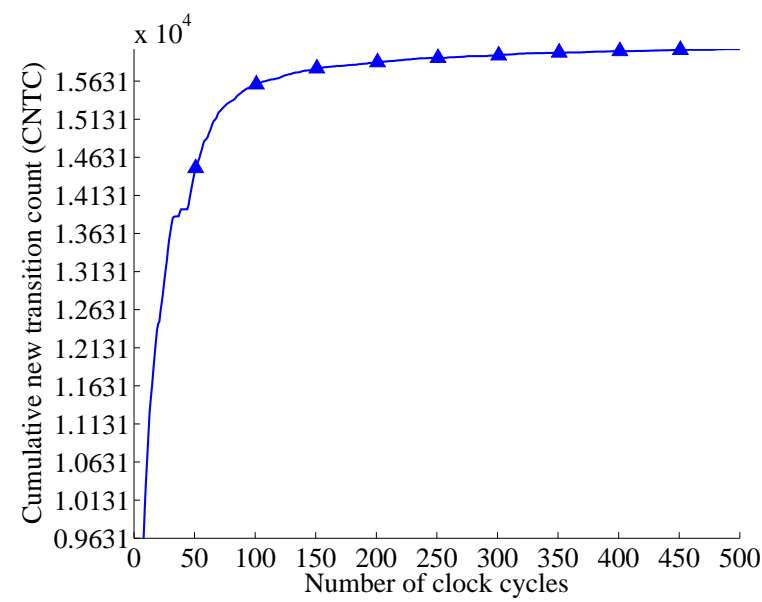

Fig. 2. An example to illustrate cumulative new transition counts.

Here th is the threshold value for the design.

Suppose $T S$ is composed of $q$ instructions $I_{1}, I_{2} \ldots, I_{q}$. For each instruction $I_{j}$, suppose the countable $0 \rightarrow 0 \mathrm{TC}$ of register $R_{i}(1 \leq i \leq k)$ for instruction $I_{j}$ is $t_{i j 00}^{*}$, the countable $0 \rightarrow 1 \mathrm{TC}$ of register $R_{i}$ for $I_{j}$ is $t_{i j 01}^{*}$, the countable $1 \rightarrow 0 \mathrm{TC}$ of register $R_{i}$ for $I_{j}$ is $t_{i j 10}^{*}$, and the countable $1 \rightarrow 1 \mathrm{TC}$ of register $R_{i}$ for $I_{j}$ is $t_{i j 11}^{*}$. Given the threshold value $t h$, the output deviation $\Delta(j)$ for instruction $I_{j}$ can be calculated by considering all possible countable transitions and the different registers:

$$
\begin{aligned}
& \Delta(j)=\frac{1}{k} \cdot \frac{1}{4 \cdot \max _{i=1}^{k}\left\{t h \cdot o_{i} \cdot w_{i}\right\}}\left(\sum_{i=1}^{k} t_{i j 00}^{*} \cdot o_{i} \cdot w_{i}+\right. \\
& \left.\sum_{i=1}^{k} t_{i j 01}^{*} \cdot o_{i} \cdot w_{i}+\sum_{i=1}^{k} t_{i j 10}^{*} \cdot o_{i} \cdot w_{i}+\sum_{i=1}^{k} t_{i j 11}^{*} \cdot o_{i} \cdot w_{i}\right)
\end{aligned}
$$

In Equation (1), $\sum_{i=1}^{k} t_{i j 00}^{*} \cdot o_{i} \cdot w_{i}$ represents the contribution of countable $0 \rightarrow 0$ transitions of all registers. Similarly, $\sum_{i=1}^{k} t_{i j 01}^{*} \cdot o_{i} \cdot w_{i}, \sum_{i=1}^{k} t_{i j 10}^{*} \cdot o_{i} \cdot w_{i}$, and $\sum_{i=1}^{k} t_{i j 11}^{*} \cdot o_{i} \cdot w_{i}$ represent the contribution of countable $0 \rightarrow 1,1 \rightarrow 0$ and $1 \rightarrow 1$ transitions of all registers, respectively. Based on the deviation definition in Section II, the deviation for $T S$ can be calculated as

$$
\begin{aligned}
\Delta^{*}(T S)= & \sum_{j=1}^{q} \Delta(j) \\
= & \frac{1}{4 k \cdot \max _{i=1}^{k}\left\{t h \cdot o_{i} \cdot w_{i}\right\}} \cdot \\
& \sum_{j=1}^{q} \sum_{i=1}^{k}\left\{\left(t_{i j 00}^{*}+t_{i j 01}^{*}+t_{i j 10}^{*}+t_{i j 11}^{*}\right) \cdot o_{i} \cdot w_{i}\right\} \\
= & \frac{1}{4 k \cdot \max _{i=1}^{k}\left\{t h \cdot o_{i} \cdot w_{i}\right\}} \cdot \\
& \sum_{i=1}^{k}\left\{\sum_{j=1}^{q}\left\{\left(t_{i j 00}^{*}+t_{i j 01}^{*}+t_{i j 10}^{*}+t_{i j 11}^{*}\right) \cdot o_{i} \cdot w_{i}\right\}\right\}
\end{aligned}
$$
These contributions are normalized by $4 \cdot \max _{i=1}^{k}\left\{t h \cdot o_{i} \cdot w_{i}\right\}$.

$S_{i 10}=\sum_{j=1}^{q}\left\{t_{i j 10}^{*} \cdot o_{i} \cdot w_{i}\right\}$, and $S_{i 11}=\sum_{j=1}^{q}\left\{t_{i j 11}^{*} \cdot o_{i} \cdot w_{i}\right\}$, Equation (2) can now be written as

$$
\begin{aligned}
\Delta^{*}(T S)= & \frac{1}{4 k \cdot \max _{i=1}^{k}\left\{t h \cdot o_{i} \cdot w_{i}\right\}} \\
& \sum_{i=1}^{k}\left\{S_{i 00}+S_{i 01}+S_{i 10}+S_{i 11}\right\}
\end{aligned}
$$

Note that $S_{i 00}$ represents the aggregated contributions of countable $0 \rightarrow 0$ TC of register $R_{i}$ for all the instructions in $T S$. The parameters $S_{i 01}, S_{i 10}$, and $S_{i 11}$ are defined in a similar way.

Let $S_{00}^{*}=\sum_{i=1}^{k} S_{i 00}, S_{01}^{*}=\sum_{i=1}^{k} S_{i 01}, S_{10}^{*}=\sum_{i=1}^{k} S_{i 10}$, and $S_{11}^{*}=\sum_{i=1}^{k} S_{i 11}$, Equation (3) can be rewritten as:

$$
\begin{aligned}
\Delta^{*}(T S)= & \frac{1}{4 k \cdot \max _{i=1}^{k}\left\{t h \cdot o_{i} \cdot w_{i}\right\}} . \\
& \left\{S_{00}^{*}+S_{01}^{*}+S_{10}^{*}+S_{11}^{*}\right\}
\end{aligned}
$$

Note that $S_{00}^{*}$ represents the aggregated contributions of countable $0 \rightarrow 0$ TC of all registers for all the instructions in $T S$. The parameters $S_{01}^{*}, S_{10}^{*}$, and $S_{11}^{*}$ are defined in a similar way.

A major difference here compared to [27] lies in the manner in which RTL output deviations are computed. In [27], a confidence-level measure is used for deviation calculation. This probabilistic measure is hard to obtain since it depends on layout and failure data. Moreover, it is difficult to justify the particular values used for deviation computation. In contrast, the CNTC measure in this work is easy to obtain through a pre-processing of a subset of functional test sequences, and it can be related to the unique transitions in the registers of the design.

\section{EXPERIMENTAL RESULTS}

We next evaluate the efficiency of deviation-based testsequence grading by performing experiments on the Biquad filter core [18] and the Scheduler module of the Illinois Verilog Model (IVM) [19] [20]. The Biquad filter core is an implementation of an infinite impulse response (IIR) filter with two poles and two zeros. It uses the wishbone interface for reading and writing of filter coefficient registers. The coefficient width is set to 64 bits. A synthesized gate-level design for the Biquad filter consists of 160,147 gates and 1116 flip-flops. IVM employs a microarchitecture that is similar in complexity to the Alpha 21264. It has most of the features of modern microprocessors, featuring superscalar operation, dynamical scheduling, and out-of-order execution. Relevant research based on IVM has recently been reported in [21] [22]. The Verilog model for Scheduler consists of 1090 lines of code. A synthesized gate-level design for it consists of 375,061 gates and 8,590 flip-flops.

Our first goal is to show high correlation between RT-level deviations and gate-level coverage for various fault models. The second goal is to investigate the ramp-up of the gate-level fault coverage for various functional test sequences. These sequences are obtained using different sequence-reordering methods. It is well-known that we cannot accurately evaluate

(2) unmodeled defect coverage through simulation using a specific Let $S_{i 00}=\sum_{j=1}^{q}\left\{t_{i j 00}^{*} \cdot o_{i} \cdot w_{i}\right\}, S_{i 01}=\sum_{j=1}^{q}\left\{t_{i j 01}^{*} \cdot o_{i} \cdot w_{i}\right\}, \quad$ fault model, because this approach relies on the fault model to 
represent defect behavior. Unmodeled defect coverage can be determined accurately by analyzing silicon data. In this work, we consider various fault models to represent defect behavior and evaluate the correlation between RTL deviations and gatelevel coverage for each fault model.

\section{A. Results for Biquad filter}

1) Experimental setup: All experiments for Biquad filter were performed on a 64-bit Linux server with 4 GB memory. Design Compiler (DC) from Synopsys was used to extract the gate-level information for calculating weight vector. Synopsys Verilog Compiler (VCS) was used to run Verilog simulation and compute the deviations. A commercial tool was used to run gate-level fault simulation and Matlab was used to obtain the Kendall's correlation coefficient [23]. The Kendall's correlation coefficient is used to measure the degree of correspondence between two rankings and assessing the significance of this correspondence. It is used in this paper to measure the degree of correlation of RT-level deviations and gate-level fault coverage. A coefficient of 1 indicates perfect correlation while a coefficient of 0 indicates no correlation.

We adopt 6 test sequences, labeled as $T S_{1}, T S_{2}, \ldots, T S_{6}$, respectively. Each sequence consists of 500 clock cycles of vectors. $T S_{1}, T S_{2}$ and $T S_{3}$ are generated according to the functionality of the Biquad filter. The data input in $T S_{1}, T S_{2}$ and $T S_{3}$ is composed of samples from a sinusoid signal with $1 \mathrm{KHz}$ frequency, a cosine signal with $15 \mathrm{KHz}$ frequency and white noise. In $T S_{4}, T S_{5}$ and $T S_{6}$, the data input is randomly generated.

2) Threshold value: In order to calculate the RT-level output deviations, we need to set the threshold value. We obtain the threshold value for Biquad filter according to the description in Section III. In this work, we randomly select two test sequences, $T_{1}$ and $T_{4}$, for learning the threshold value. For each of them, we run Verilog simulation and draw the figure of cumulative new transition counts, as shown in Figure 3. We can obtain the critical points for $T_{1}$ and $T_{4}$ are 242th, 228 th clock cycle respectively. We then record the aggregated TCs for all registers till the critical point for each of the two target test sequences, i.e., (270072 254448). Since there are altogether 17 registers in the Biquad filter, we set the threshold value $T H \_1$ to be $(270072 / 17+254448 / 17) / 2$, i.e, 15,427 . In the above setting of threshold value, the CNTC of the critical point is $98 \%$ of the maximum CNTC. We can also consider other percentages in setting the critical point. For example, for a given percentage of $95 \%$, we obtain the threshold value as 7,878 , labeled as $T H \_2$. For a percentage of $90 \%$, we obtain the threshold value as 4,759 , labeled as $T H \_3$.

3) Correlation between output deviations and gate-level fault coverage: For these six functional test sequences, we derived the Kendall's correlation coefficient between their RTlevel output deviations and their gate-level fault coverage. The stuck-at fault model is used for the Biquad filter, as well as the transition fault model, the bridging-coverage estimate $(B C E)$ [25], and the modified $B C E$ measure $\left(B C E^{+}\right)$[26]. Figure 4 presents details about the experimental flow. First we calculate the deviations for the six functional test sequences according

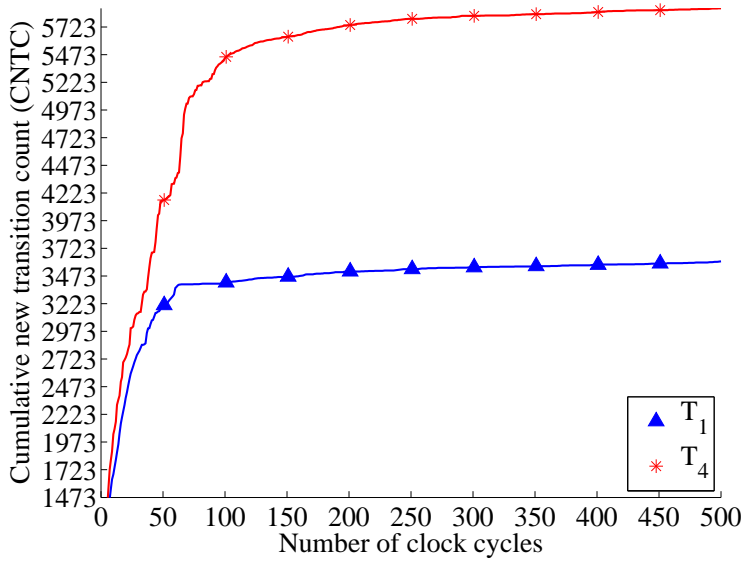

Fig. 3. Cumulative new transition counts for $T_{1}$ and $T_{4}$ (Biquad filter).

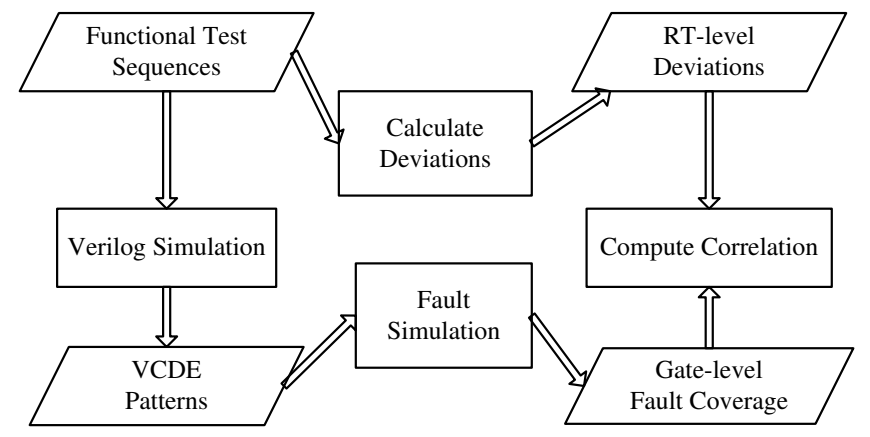

Fig. 4. Experimental flow.

to the method described in Section III . For each threshold value, we obtain the deviation values for the six functional test sequences and record them as a vector. For example, for the threshold value $T H_{-} 1$, the deviations are recorded as $D E V_{-} 1\left(\operatorname{dev}_{1}, \operatorname{dev}_{2}, \ldots, \operatorname{dev}_{6}\right)$.

We next obtain test patterns in extended-VCD (VCDE) format for the six functional test sequences by running Verilog simulation. Using the VCDE patterns, gate-level fault coverage is obtained by running fault simulation for the ten functional test sequences. The coverage values are recorded as a vector: $\operatorname{COV}\left(\operatorname{cov}_{1}, \operatorname{cov}_{2}, \ldots, \operatorname{cov}_{6}\right)$.

In order to evaluate the effectiveness of the deviation-based functional test-grading method, we calculate the Kendall's correlation coefficient between $D E V \_1$ and $C O V$. Figure 5 shows the correlation between deviations and stuckat/transition fault coverage, as well as between deviations and $B C E / B C E^{+}$metrics, for different threshold values. For stuck-at faults and bridging faults, we see that the coefficients are very close to 1 for all three threshold values. For transition faults, the correlation is less, but still significant. The results demonstrate that the deviation-based method is effective for grading functional test sequences. The total CPU time for deviation calculation and test-sequence grading is less than 2 hours. The CPU time for gate-level stuck-at (transition) fault simulation is 12 (19) hours, and the CPU time for computing the gate-level BCE (BCE+) measure is 15 (18) hours, thus we are able to reduce CPU time significantly.

Note that we have proposed the use of weight and observ- 
ability vectors to improve the accuracy of fault grading. If observability and weight vectors are ignored and only transition counts are considered in the calculation of output deviations, the correlation coefficients between output deviations and gatelevel fault coverage are reduced considerably. These results are shown in Figure 6.

4) Cumulative gate-level fault coverage (Ramp-up): We next evaluate the effectiveness of output deviations by comparing the cumulative gate-level fault coverage of several reordered functional test sequences. For the Biquad filter, traditional stuck-at fault coverage as well as $B C E+$ metric are considered.

These reordered sets are obtained in four ways: (i) baseline order $T S_{1}, T S_{2} \ldots, T S_{6}$; (ii) random ordering $T S_{1}, T S_{3}, T S_{2}, T S_{6}, T S_{4}, T S_{5}$; (iii) random ordering $T S_{3}, T S_{5}, T S_{1}, T S_{2}, T S_{4}, T S_{6}$; (iv) the descending order of output deviations. In the deviation-based method, test sequences with higher deviations are ranked first.

Figure 7-8 show cumulative stuck-at fault coverage, and $B C E+$ metric for the four reordered functional test sequences following the above four orders. We can see that the deviationbased method results in the steepest curves for cumulative stuck-at coverage and $B C E+$ metric.

5) Correlation results for longer functional test sequences: The above results are obtained based on 6 test sequences, each

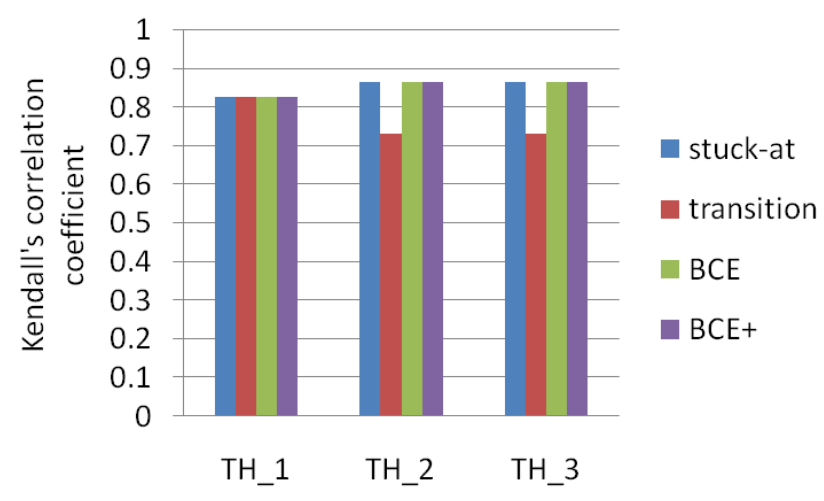

Fig. 5. Correlation between output deviations and gate-level fault coverage (Biquad).

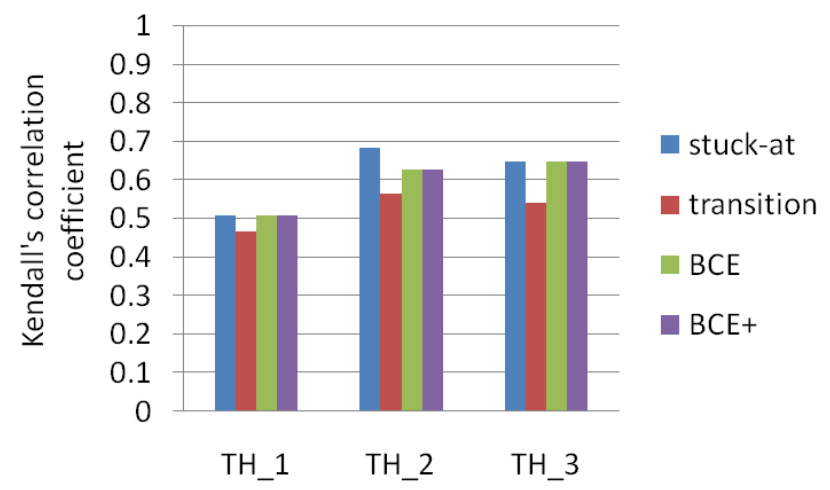

Fig. 6. Correlation between output deviations (only consider transition counts) and gate-level fault coverage (Biquad).

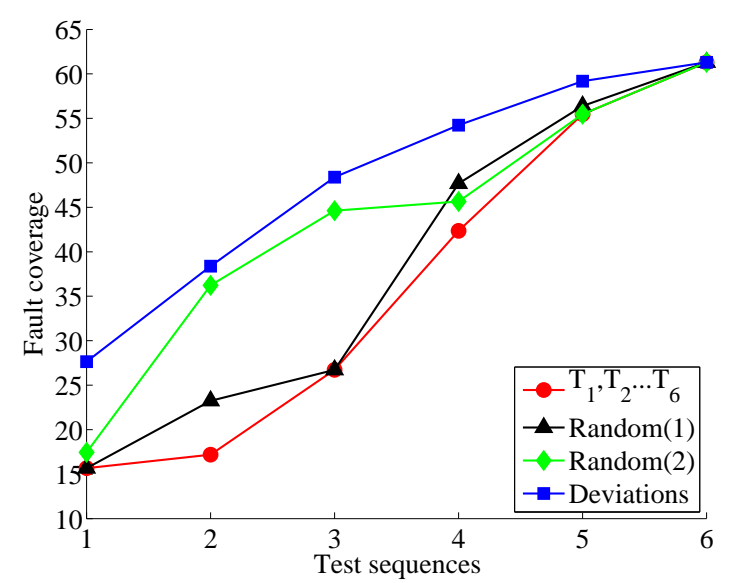

Fig. 7. Cumulative stuck-at fault coverage for various test-sequence ordering methods (Biquad).

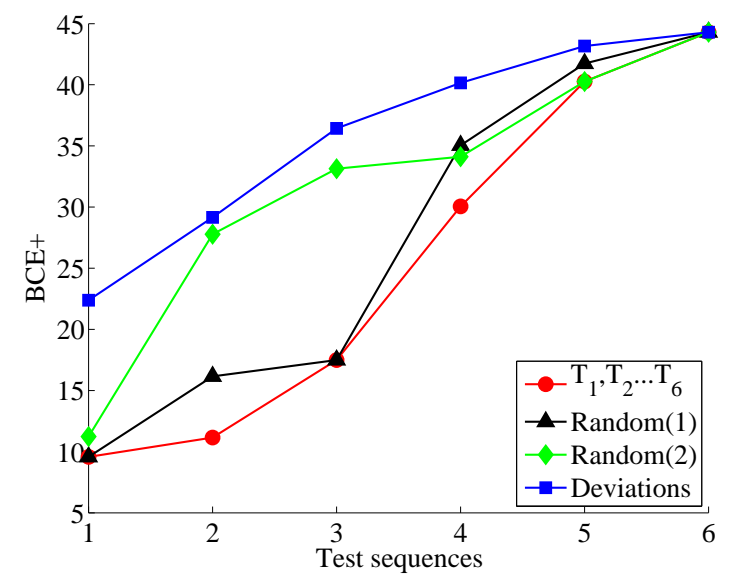

Fig. 8. Cumulative BCE+ for various test-sequence ordering methods (Biquad).

of which consists of 500 clock cycles. To further evaluate the effectiveness of our method, we consider longer functional test sequences. Each test sequence is now 5,000 clock cycles long. The data inputs in $T S_{1}, T S_{2}$ and $T S_{3}$ are obtained by changing the sampling frequency so that they are composed of 5,000 cycles. For $T S_{4}, T S_{5}$ and $T S_{6}$, the data input is randomly generated.

Figure 9 shows the correlation between deviations and stuck-at/transition fault coverage, as well as between deviations and $B C E / B C E+$ metrics, for different threshold values, namely $98 \%, 95 \%$, and $90 \%$. We can see that the coefficients are still significant for all three threshold values. The results demonstrate that the deviation-based method is effective for grading longer functional test sequences.

\section{B. Results for Scheduler module}

To further demonstrate the benefits of the RT-level deviation metric for larger circuits and longer test sequences, we perform experiments on the Scheduler module.

The experimental setup and procedure are similar to that of Biquad filter: 1) obtain the RT-level deviations by considering TCs, observability vector, weight vector and threshold value; 


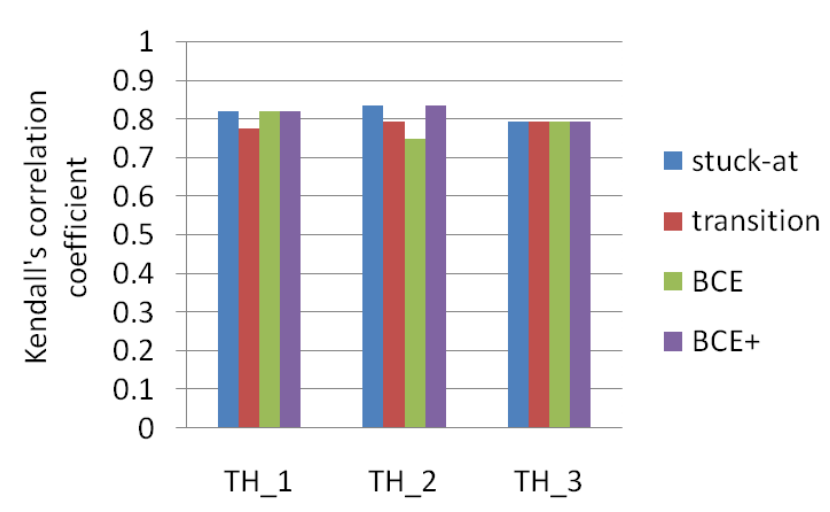

Fig. 9. Correlation result for longer functional test sequences (Biquad).

TABLE II

REGISTERS AND REGISTER ARRAYS IN THE SCHEDULER MODULE.

\begin{tabular}{|r|r|r||r|r|r|}
\hline Name & Width & Array & Name & Width & Array \\
\hline sb_counters & 3 & $0: 79$ & instout0 & 222 & - \\
\hline issued & 1 & $0: 31$ & instout1 & 222 & - \\
\hline valid & 1 & $0: 31$ & instout2 & 222 & - \\
\hline issue_head & 5 & - & instout3 & 222 & - \\
\hline issue_tail & 5 & - & instout4 & 222 & - \\
\hline inst_array & 217 & $0: 31$ & instout5 & 222 & - \\
\hline
\end{tabular}

2) obtain the gate-level fault coverage; 3 ) calculate the correlation coefficient and compare the coverage ramp-up curves.

1) Scheduler module: The Scheduler dynamically schedules instructions, and it is a key component of the IVM architecture [21]. It contains an array of up to 32 instructions waiting to be issued and can issue 6 instructions in each clock cycle.

Table II shows the registers and register arrays in the Scheduler module. The first and the fourth columns list the name of the register or register array. The second and fifth columns represent the width of each register in bits. The third and sixth columns indicate whether it is a register array. If it is a register array, the index value is shown.

2) Experimental setup: The experimental setup is the same as that for Biquad filter. For the Scheduler module, all experiments are based on 10 functional test sequences. Six of them are composed of instruction sequences, referred to as $T_{0}, T_{1}, T_{2}, T_{3}, T_{4}, T_{5}$. The other four, labeled as $T_{6}, T_{7}$, $T_{8}$ and $T_{9}$, are obtained indirectly by sequential ATPG. First, cycle-based gate-level stuck-at ATPG is carried out. From the generated patterns, stimuli are extracted and composed into a functional test sequence, labeled as $T_{6}$. Next we perform "not", "xnor", "xor" bit-operations on $T_{6}$ separately and obtain new functional test sequences $T_{7}, T_{8}$ and $T_{9} . T_{7}$ is obtained by inverting each bit of $T_{6} ; T_{8}$ is obtained by performing "xnor" operation between the adjacent bits of $T_{6} ; T_{9}$ is obtained by performing "xor" operation between the adjacent bits of $T_{6}$. The above bit-operations do not affect the clock and reset signals in the design.

3) RT-level deviations: Using the deviation-calculation method in Section III, we calculate the RT-level deviations for the Scheduler module by considering the parameter TC, weight vector, observability vector and threshold value.
TABLE III

WEIGHT VECTOR FOR SCHEDULER MODULE.

\begin{tabular}{|r|r|r|r|r|}
\hline Registers & $\begin{array}{r}\text { Fanin } \\
\text { count }\end{array}$ & $\begin{array}{r}\text { Fanout } \\
\text { count }\end{array}$ & $\begin{array}{r}\text { No. of } \\
\text { connections }\end{array}$ & Weight \\
\hline sb_counters & 9211 & 4800 & 14011 & 0.0183 \\
\hline issued & 112932 & 355464 & 468396 & 0.6113 \\
\hline valid & 4886 & 355482 & 360368 & 0.4703 \\
\hline issue_head & 2986 & 397086 & 400072 & 0.5221 \\
\hline issue_tail & 146 & 202732 & 202878 & 0.2648 \\
\hline inst_array & 275639 & 490584 & 766223 & 1 \\
\hline instout0 & 92962 & 4367 & 97329 & 0.1270 \\
\hline instout1 & 118629 & 4367 & 122996 & 0.1605 \\
\hline instout2 & 92553 & 4775 & 97328 & 0.1270 \\
\hline instout3 & 92638 & 4687 & 97325 & 0.1270 \\
\hline instout4 & 93045 & 4137 & 97182 & 0.1268 \\
\hline instout5 & 118337 & 4137 & 122474 & 0.1598 \\
\hline
\end{tabular}

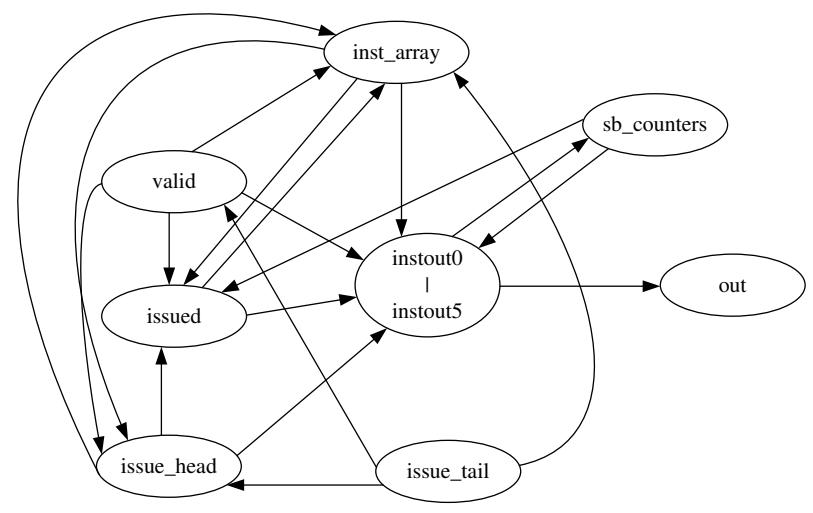

Fig. 10. Dataflow diagram of Scheduler module.

To obtain the weight vector, we first determine the fanin and fanout counts for each register or register array. A net is considered to be in the fanin cone of a register if there is a path through combinational logic from the net to that register. Similarly, a net is considered to be in the fanout cone of a register if there is a path through combinational logic from that register to the net. Table III lists these counts for each register (array) and shows the weight vector components. In Column 4, "No. of connections" is the sum of the fanin count and the fanout count.

The observability vector is obtained using the dataflow diagram extracted from the design. The dataflow diagram is extracted automatically. Many logic analysis tools can implement this function. Here, we use Design Compiler of Synopsys and use its features of tracing fanin and fanout registers to construct the dataflow graph. Figure 10 shows the dataflow diagram of the Scheduler module.

We consider the following order of the registers: sb_counters, issued, valid, issue_head, issue_tail, instout 0 , instout1, instout2, instout3, instout4, instout5, and inst_array. The corresponding observability vector is (0.5 0.50 .50 .333311111110 .5$)$.

We obtain the threshold value according to the description in Section III. In this work, we randomly select four test sequences, $T_{0}, T_{1}, T_{6}, T_{7}$, for learning the threshold value. For each of them, we run Verilog simulation and generate the graph of cumulative new transition counts, as shown in Figures 11-12. We obtain the critical points for $T_{0}, T_{1}, T_{6}, T_{7}$ are 


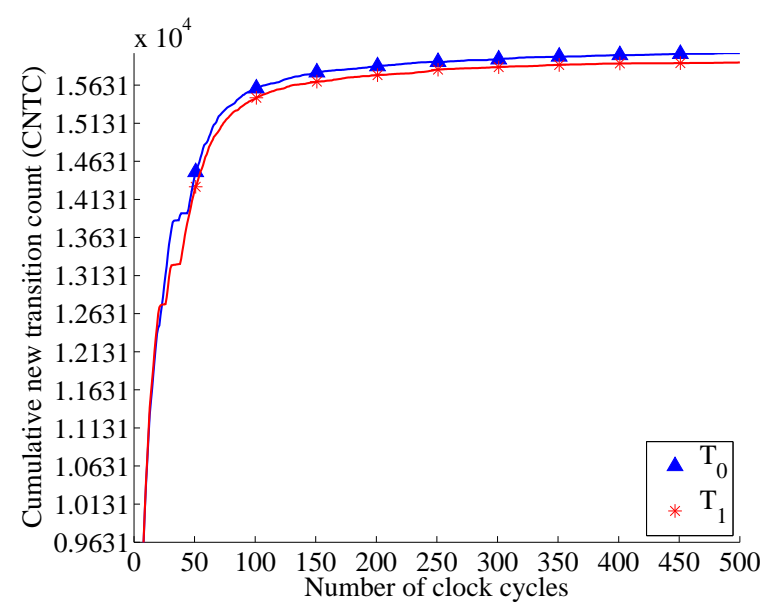

Fig. 11. Cumulative new transition counts for $T_{0}$ and $T_{1}$ (Scheduler module).

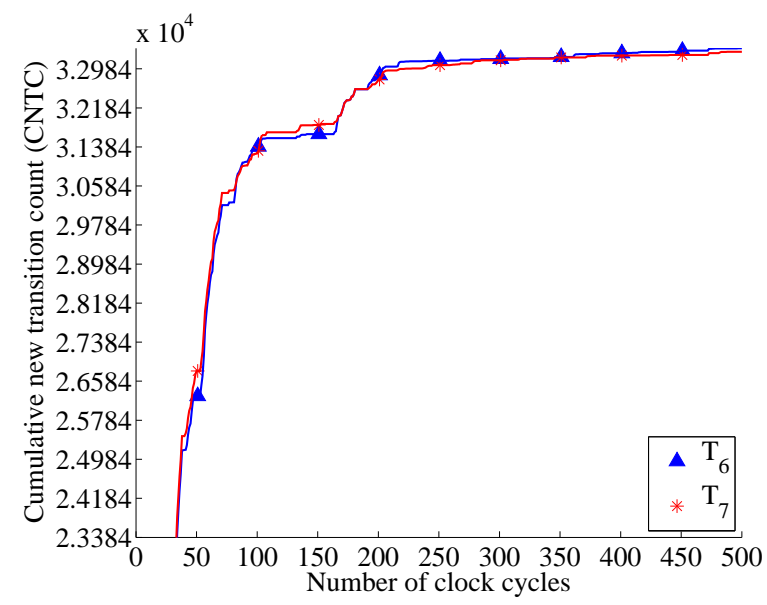

Fig. 12. Cumulative new transition counts for $T_{6}$ and $T_{7}$ (Scheduler module).

130th, 126th, 195th, and 195th clock cycle, respectively. We then record the aggregated TCs for all registers at the critical point for each of the four target test sequences, i.e., (1116700 $108234016750501675050)$. Since there are altogether 12 registers in the Scheduler module, we set the threshold value TH_1 to be $(1116700 / 12+1082340 / 12+1675050 / 12+$ $1675050 / 12) / 4$, i.e, 115,610 . In the above setting of threshold value, the CNTC of the critical point is $98 \%$ of the maximum CNTC. We can also consider other percentage when obtaining critical point. For example, for a given percentage of $95 \%$, we obtain the threshold value as 74,447 , labeled as $T H \_2$. For a percentage of $90 \%$, we obtain the threshold value as 43,129 , labeled as TH_3.

Given the information of the TCs, the weight vector, the observability vector and the threshold value, RT-level deviations can be calculated. The corresponding RT-level deviations for the Scheduler module are shown in Table IV (FT stands for functional test). The total CPU time for deviation calculation and test-sequence grading is less than 8 hours. The CPU time for gate-level stuck-at (transition) fault simulation is 110 (175) hours and the CPU time for computing the gate-level $B C E$ $\left(B C E^{+}\right)$measure is $120(125)$ hours, thus we are able to

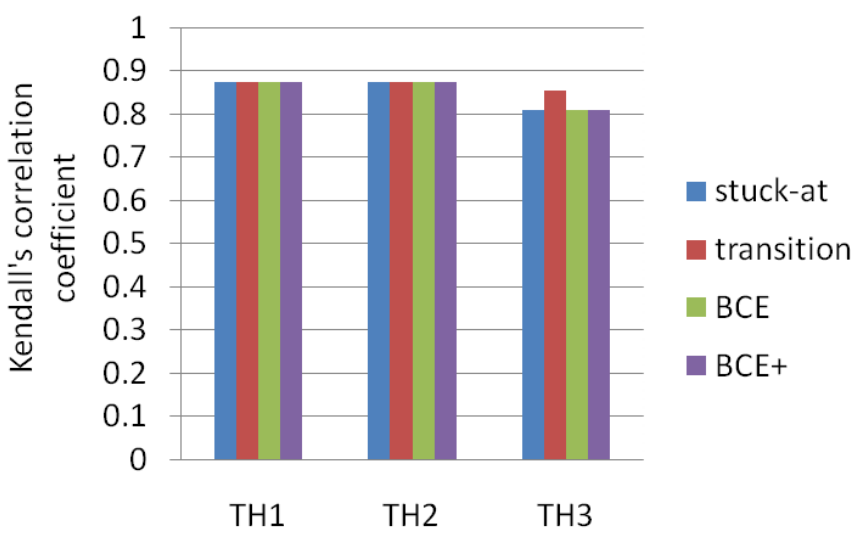

Fig. 13. Correlation between output deviations and gate-level fault coverage (Scheduler module).

reduce CPU time by over an order of magnitude.

4) Correlation between output deviations and gate-level fault coverages: We obtain the stuck-at and transition fault coverages for the functional test sequences by running fault simulation at the gate-level. Bridging fault coverage is estimated using the $B C E$ and $B C E^{+}$metrics. The correlation between these gate-level fault coverage measures and RTlevel deviations are computed and the Kendall's correlation coefficients are shown in Figure 13. As in the case of the Biquad filter, the correlation coefficients are close to 1 for all the three threshold values. This demonstrates that the RT-level deviations are a good predictor of the gate-level fault coverage.

As in the case of the Biquad filter, if observability and weight vectors are ignored and only transition counts are considered in the calculation of output deviations, we observe a considerable degradation in the correlation coefficients between output deviations and gate-level fault coverage. These results are shown in Figure 14.

5) Cumulative gate-level fault coverage (Ramp-up): We evaluate the cumulative gate-level fault coverage of several reordered functional test sequences. Besides traditional stuck-

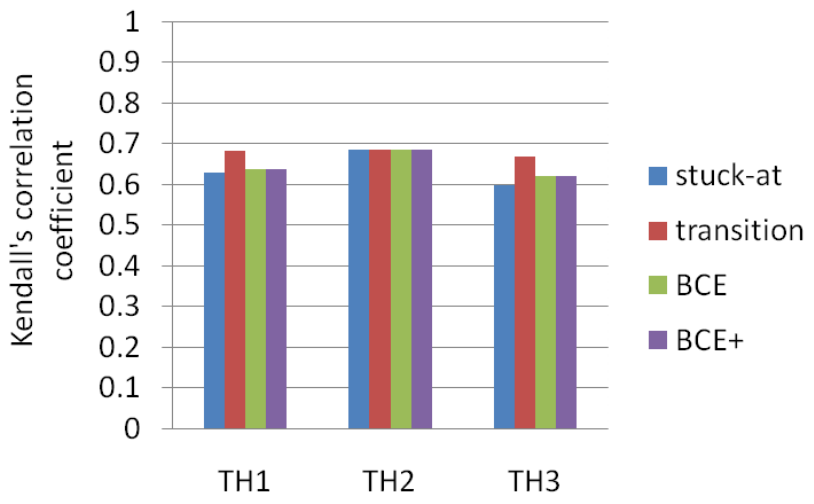

Fig. 14. Correlation between output deviations (only consider transition counts) and gate-level fault coverage (Scheduler module). 
TABLE IV

RT-LEVEL DEVIATIONS OF TEN FUNCTIONAL TESTS FOR SCHEDULER MODULE.

\begin{tabular}{|c|c|c|c|c|c|c|c|c|c|c|}
\hline Functional test & \multirow{2}{*}{$T_{0}$} & $T_{1}$ & $T_{2}$ & $T_{3}$ & $T_{4}$ & $T_{5}$ & $T_{6}$ & $T_{7}$ & $T_{8}$ & $T_{9}$ \\
\hline Threshold value & 0.3324 & 0.3324 & 0.3304 & 0.3308 & 0.3320 & 0.3324 & 0.4220 & 0.4256 & 0.3652 & 0.3680 \\
\hline$T H \_1$ & 0.3424 & 0.3424 & 0.3400 & 0.3408 & 0.3420 & 0.3424 & 0.4324 & 0.4364 & 0.3772 & 0.3804 \\
\hline$T H \_2$ & 0.3480 & 0.3476 & 0.3456 & 0.3464 & 0.3472 & 0.3476 & 0.4380 & 0.4420 & 0.3840 & 0.3868 \\
\hline$T H \_3$ &
\end{tabular}

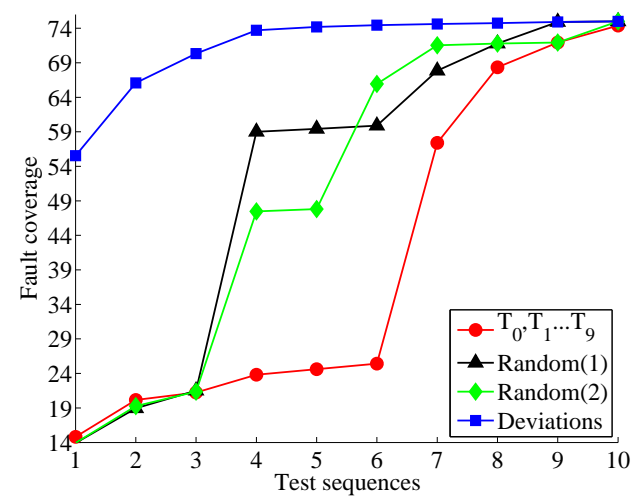

Fig. 15. Cumulative stuck-at fault coverage for various test-sequence ordering methods (Scheduler module).

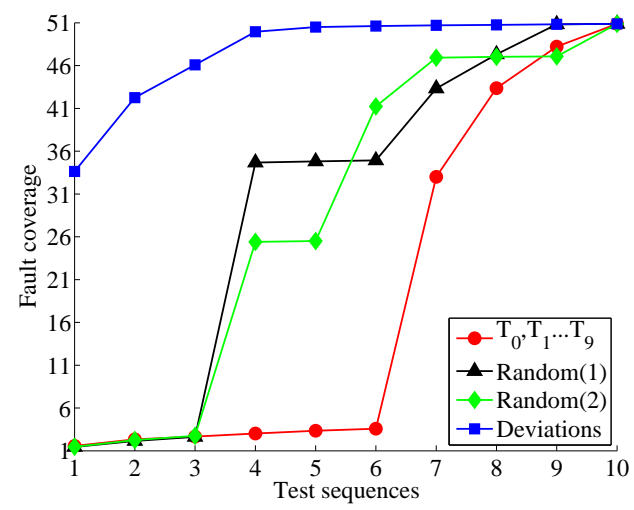

Fig. 16. Cumulative transition fault coverage for various test-sequence ordering methods (Scheduler module).

at and transition fault coverage, we use $B C E+$ metric to evaluate unmodeled defect coverage.

These reordered sequences sets are obtained in four ways: (i) baseline order $T_{0}, T_{1} \ldots, T_{9}$; (ii) random ordering $T_{2}, T_{1}, T_{5}, T_{7}, T_{0}, T_{3}, T_{8}, T_{9}, T_{6}, T_{4}$; (iii) random ordering $T_{4}, T_{1}, T_{0}, T_{9}, T_{2}, T_{7}, T_{6}, T_{3}, T_{5}, T_{8}$; (iv) the descending order of output deviations. In the deviation-based method, test sequences with higher deviations are ranked first. Figure 1517 show cumulative stuck-at, transition fault coverages and $B C E+$ for the four reordered functional test sequences following the above four orders. We find that the deviation-based method results in the steepest cumulative stuck-at, transition and bridging fault coverage curves.

\section{Comparison of proposed method with [11]}

A method based on traversed circuit states was proposed recently to estimate the gate-level fault coverage of functional

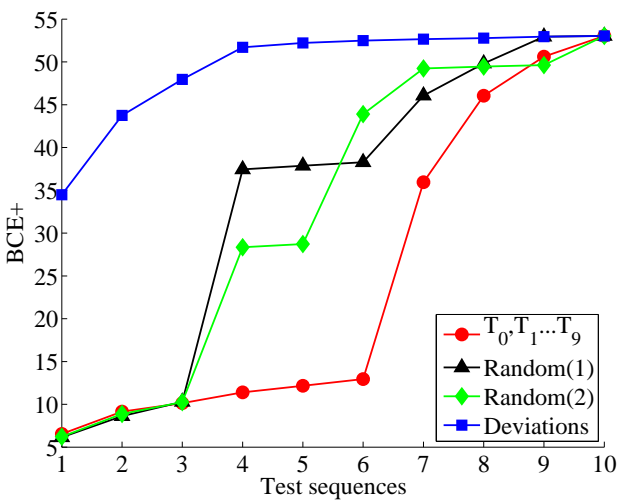

Fig. 17. Cumulative $B C E+$ metric for various test-sequence ordering methods (Scheduler module).

test sequences [11]. We consider [11] as a baseline and compare it to the results obtained by the proposed method.

Based on gate-level fault coverage, we can obtain an ordering of the given functional test sequences. We consider this order to be golden (desired) ordering. Based on the RT-level output deviations and the estimated gate-level fault coverage as in reference [11], we obtain two other orders of these sequences, labeled as Order_dev and Order_Ref[11]. Our goal is to observe whether Order_dev has more similarity with the golden order compared to Order_Ref[11]. The problem of comparing the similarity of two orders can be viewed as a sequence-matching problem. The sequencematching problem has been extensively studied in the literature [28] [29]. We choose a widely used metric, namely the longest common subsequence (LCS), to evaluate the similarity of two sequences.

In our problem, we can simply calculate the common subsequence starting with every functional test sequence, and then choose the longest common subsequence as the LCS of the orders. For example, suppose two orders to be compared are $T S 1>T S 2>T S 3>T S 4>T S 5$ and $T S 2>T S 3>$ $T S 4>T S 1>T S 5$. There are no common subsequences starting with $T S 1, T S 4$ and TS5. The common subsequence starting with $T S 2$ is $T S 2>T S 3>T S 4$; the common subsequence starting with $T S 3$ is $T S 3>T S 4$. Therefore, the LCS of these two orders is TS2 > TS3 $>T S 4$, and the length of the LCS is 3.

Table V compares the proposed method to [11] based on the length of the LCS. We can see that the length of LCS for Order_dev and the golden order based on stuck-at fault coverage is 4, while the length of LCS for Order_Ref[11] and the golden order based on stuck-at fault coverage is 3 . In other words, the ordering based on the proposed method 
TABLE V

LENGTH OF LCS.

\begin{tabular}{|r|r|r|}
\hline Order & Order_dev & Order_Ref $[11]$ \\
\hline Stuck-at fault coverage & 4 & 3 \\
\hline Transition fault coverage & 4 & 2 \\
\hline$B C E+$ & 3 & 3 \\
\hline
\end{tabular}

has more similarity with the golden order. Similar results are obtained when we consider the transition fault model. For the $B C E+$ measure, the LCS length for the two methods are the same. This implies that the proposed method is more effective in grading functional test sequences than [11]. The reason may lie in the fact that observability of registers is considered in the proposed method but it is not considered in reference [11]. Since observability is an important factor that affects fault coverage, the proposed method can provide a more effective ordering of functional test sequences.

Another advantage of the proposed method is that it is scalable to large designs since it requires less computation time. The total CPU time for deviation calculation and testsequence grading for the Scheduler module is less than 8 hours. On the other hand, the CPU time for grading test sequences based on [11] is about 73 hours. This is because the method in [11] requires logic simulation of the gatelevel circuit. Therefore, [11] is not scalable for more realistic scenarios, where more functional test sequences have to be considered. Furthermore, with the proposed RT-level deviation based method, we are able to reduce CPU time by nearly a order of magnitude, with better or comparable test-sequence grading results.

\section{Results for different gate-level implementations}

There can be different gate-level implementations for the same RT-level design. A gate-level netlist is usually obtained by applying synthesis procedure to a given RT-level design. Different technology libraries or synthesis constraints such as timing and area requirements will lead to different gatelevel netlists for the same RT-level design. For the Scheduler module, all the experiments reported thus far were carried out using a specific gate-level netlist, which we call imp1. We next evaluate whether the proposed RT-level deviationbased functional test grading method is sensitive to gate-level implementation. We first obtain two other gate-level netlists, namely $i m p 2$ and $i m p 3$, for the given Scheduler module. Note that Design Compiler (DC) from Synopsys was used in synthesis procedure to obtain the gate-level netlists. Next, we re-calculate their RT-level output deviations and observe the correlations between deviations with gate-level fault coverage metrics.

Table VI shows the comparison for these three implementations. Note that $i m p 1$ and $i m p 2$ adopt the FREEPDK library in synthesis while $i m p 3$ uses the GSCLib3.0 library. FREEPDK is a $45 \mathrm{~nm}$ gate library provided by FreePDF and GSCLib3.0 is a $180 \mathrm{~nm}$ generic library from Cadence. With respect to the synthesis constraints, $i m p 2$ is obtained by setting map_effort to be high, while imp 1 and $i m p 3$ are obtained by setting
TABLE VI

COMPARISON OF THREE IMPLEMENTATIONS.

\begin{tabular}{|r|r|r|r|}
\hline & $i m p 1$ & $i m p 2$ & $i m p 3$ \\
\hline Technology library & FREEPDK & FREEPDK & GSCLib3.0 \\
\hline Map_effort & medium & high & medium \\
\hline No. of gates & 375,061 & 300,422 & 296,367 \\
\hline No. of flip-flops & 8,590 & 8,590 & 8,590 \\
\hline No. of stuck-at faults & $1,459,262$ & 974,298 & $1,113,422$ \\
\hline
\end{tabular}

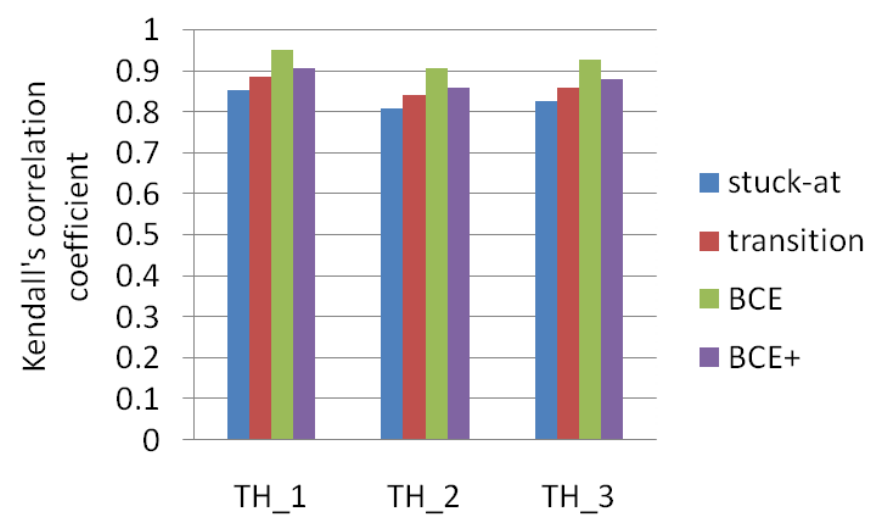

Fig. 18. Correlation between output deviations and gate-level fault coverage for $i m p 2$ (Scheduler module).

map_effort to be medium. When the option map_effort is set high, the synthesis tool spends more effort on improving the performance. The total numbers of gates, flip-flops, and stuckat faults for each implementation are also listed in Table VI.

RT-level output deviations are calculated based on transition counts, the weight vector, the observability vector and the threshold value. To calculate RT-level output deviations for $i m p 2$ and $i m p 3$, we only need to re-calculate the weight vector since transition counts, observability vector and threshold value remains unchanged for the same functional test and the same RT-level design. The re-calculated RT-level deviations under three threshold value (same as for imp1) are shown in Table VII and Table VIII. As expected, the deviations for the three implementations are different.

After we have obtained the RT-level deviations for $i m p 2$ and $i m p 3$, we calculate the gate-level fault coverage measures for them. Finally we compute the correlation between RTlevel deviations and gate-level fault coverage measures. The Kendall's correlation coefficient values are shown in Figure 18 and Figure 19. As in the case of $i m p 1$, the correlation coefficients are close to 1 for all the CL vectors for $i m p 2$ and imp3. These results highlight the fact that RT-level deviations are a good predictor of gate-level defect coverage for different gate-level implementations of an RT-level design. We therefore conclude that RT-level deviations can be used to grade functional test sequences for different gate-level implementations.

\section{E. Stratified sampling for threshold-value setting}

The threshold value is a key parameter in the calculation of RT-level output deviations. We propose to set the threshold value for a design by learning from a subset of the given 
TABLE VII

RT-LEVEL DEVIATIONS OF TEN FUNCTIONAL TESTS FOR $i m p 2$ OF SCHEDULER MODULE.

\begin{tabular}{|c|c|c|c|c|c|c|c|c|c|c|}
\hline Functional test & \multirow{2}{*}{$T_{0}$} & $T_{1}$ & $T_{2}$ & $T_{3}$ & $T_{4}$ & $T_{5}$ & $T_{6}$ & $T_{7}$ & $T_{8}$ & $T_{9}$ \\
\hline Threshold value & 0.4032 & 0.4032 & 0.4012 & 0.4016 & 0.4024 & 0.4028 & 0.4916 & 0.4960 & 0.4352 & 0.4376 \\
\hline$T H \_1$ & 0.4248 & 0.4244 & 0.4224 & 0.4232 & 0.4240 & 0.4244 & 0.5144 & 0.5180 & 0.4592 & 0.4620 \\
\hline$T H \_2$ & 0.4320 & 0.4320 & 0.4296 & 0.4304 & 0.4316 & 0.4320 & 0.5216 & 0.5252 & 0.4672 & 0.4704 \\
\hline$T H \_3$ &
\end{tabular}

TABLE VIII

RT-LEVEL DEVIATIONS OF TEN FUNCTIONAL TESTS FOR $i m p 3$ OF SCHEDULER MODULE.

\begin{tabular}{|c|c|c|c|c|c|c|c|c|c|c|}
\hline Functional test & $T_{0}$ & $T_{1}$ & $T_{2}$ & $T_{3}$ & $T_{4}$ & $T_{5}$ & $T_{6}$ & $T_{7}$ & $T_{8}$ & $T_{9}$ \\
\hline Threshold value & 0.4232 & 0.4232 & 0.4212 & 0.4216 & 0.4228 & 0.4232 & 0.5132 & 0.5164 & 0.4564 & 0.4588 \\
\hline$T H \_1$ & 0.4388 & 0.4388 & 0.4364 & 0.4372 & 0.4384 & 0.4388 & 0.5284 & 0.5324 & 0.4732 & 0.4764 \\
\hline$T H \_2$ & 0.4468 & 0.4468 & 0.4444 & 0.4452 & 0.4460 & 0.4464 & 0.5360 & 0.5400 & 0.4820 & 0.4848 \\
\hline$T H \_3$ &
\end{tabular}

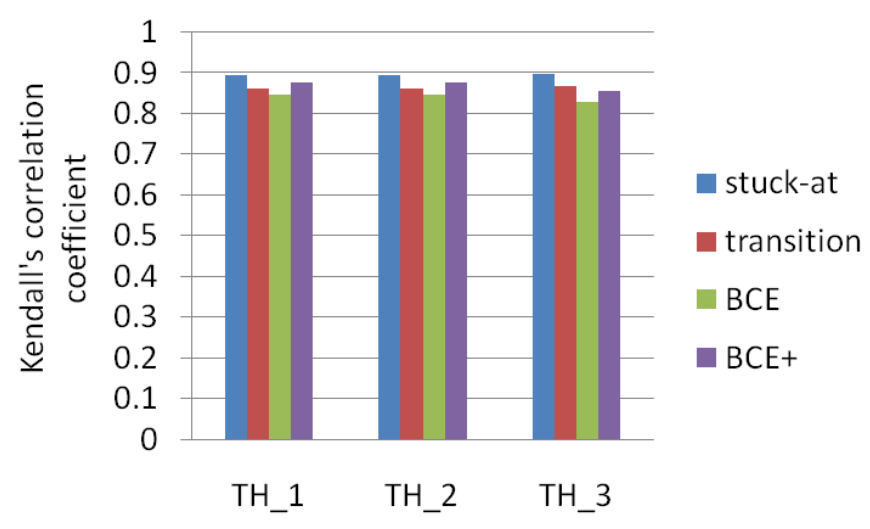

Fig. 19. Correlation between output deviations and gate-level fault coverage for imp3 (Scheduler module).

functional test sequences. However, if we randomly select several test sequences as the learning sequences, they may not represent the characteristics of the entire test set and thus may lead to inaccurate threshold values.

Here we address the problem of threshold-value setting from a survey sampling perspective [30]. The given $N$ functional test sequences constitute the population for the survey. The $C N T C$ is the characteristic under study. The threshold value is estimated by simulating the design using a number of samples drawn from the population, a procedure referred to as sampling. Our objective is to design a sampling procedure that will significantly reduce the number of simulated test sequences while ensure the accuracy of the estimated threshold value.

Stratified sampling [30] has been widely used for surveys because of its efficiency. The purpose of stratification is to partition the population into disjoint subpopulations, called strata, so that the characteristic of members within each subpopulation is more homogeneous than in the original population. In this paper, we partition the given functional test sequences into subgroups according to their sources. For the Scheduler module, we partition its 10 functional test sequences into two subgroups: Group 1 composed of 6 functional test sequences coming from Intel and Group 2 composed of 4 functional test sequences from ATPG. (To apply the stratified sampling to general designs, we may need further information about functional test sequences, such as the functionality of each test or the targeting modules of each test, to partition the given functional test sequences into appropriate subgroups.)

If the sample is taken randomly from each stratum, the procedure is known as stratified random sampling. We use stratified random sampling for the Scheduler module. For a given sample size, we first adopt random sampling for Group1 and Group2. Next we run simulation on the sampled functional test sequences and obtain the threshold value. Then based on this threshold value, we calculate the RT-level output deviations. Finally we compute the Kendall's correlation coefficient of deviation metric and gate-level fault coverage.

Table IX shows the correlation results for the Scheduler module when different stratified random sampling choices are used. The first column list the different sampling. Column 2 and Column 3 list the sample size for each subgroup. For example, for Sample 4, we sampled 3 functional test sequences from Group 1 and 2 functional test sequences from Group 2 . Columns 4-7 list the correlation coefficient of RT-level output deviations (calculated based on the sampling) and the gatelevel fault coverage. We can see that by setting threshold value using stratified sampling in the calculation of RT-level deviations, we consistently obtain good correlation results. This demonstrates the effectiveness of stratified sampling in setting threshold values. Also, we observe that in most cases, the correlation coefficient increases with the sample size. Therefore, the sample size $m$ can be chosen based on how much pre-processing can be carried out in a limited amount of time.

\section{F. Results for more functional test sequences}

To further verify the effectiveness of the RT-level output deviation metric in grading functional test sequences, we consider more functional test sequences for the Scheduler module. For the test sequences generated by sequential ATPG, we performed 3 bit-operations ("not", "xnor" and "xor") to obtain 3 new functional test sequences. Similarly, for each of the six functional test sequences provided by Intel, we 
TABLE IX

CORRELATION RESULTS WITH STRATIFIED SAMPLING (SCHEDULER MODULE).

\begin{tabular}{|c|c|c|c|c|c|c|}
\hline \multirow{2}{*}{ Sampling case } & Sample size & Sample size & \multicolumn{4}{|c|}{ Kendall's Correlation Coefficient } \\
\cline { 4 - 7 } & of Group 1 & of Group 2 & Stuck_at & Transition & $B C E$ & $B C E+$ \\
\hline 1 & 1 & 1 & 0.8540 & 0.8989 & 0.8540 & 0.8540 \\
\hline 2 & 2 & 2 & 0.8741 & 0.8741 & 0.8741 & 0.8741 \\
\hline 3 & 2 & 2 & 0.8667 & 0.8819 & 0.8540 & 0.8667 \\
\hline 4 & 3 & 2 & 0.8667 & 0.9111 & 0.8667 & 0.8741 \\
\hline 5 & 6 & 4 & 0.9080 & 0.8819 & 0.8810 & 0.8810 \\
\hline
\end{tabular}

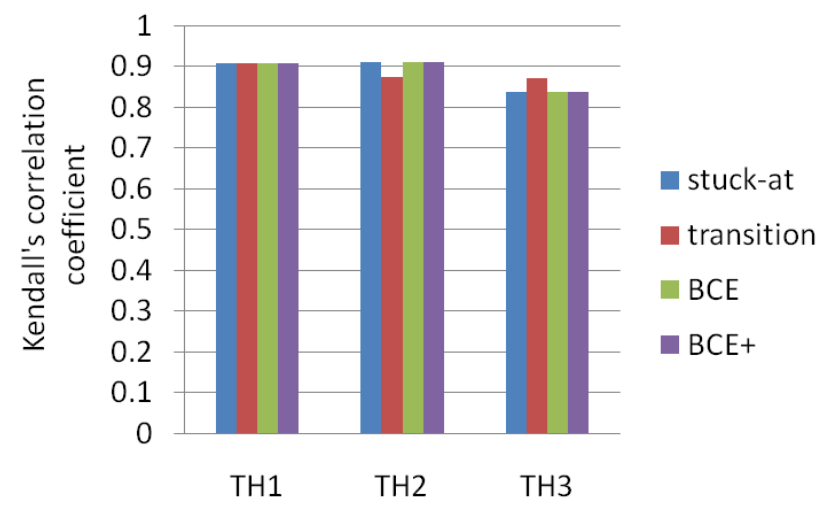

Fig. 20. Correlation results for more functional test sequences (Scheduler module).

perform the same bit-operations and obtain 3 new functional test sequences. In this way, we obtain 28 functional test sequences altogether.

We calculate the RT-level output deviations and record the gate-level fault coverage for these 28 functional test sequences. Then we compute the Kendall's correlation coefficient as in the case of 10 functional test sequences. The correlation results are shown in Figure 20. We can see that the correlation coefficient is very close to 1 . Compared to the case of 10 functional test sequences (Figure 13), the correlation is higher. This implies that our proposed method is more effective for grading a larger number of functional test sequences.

\section{Conclusions}

We have presented the output deviation metric at RT-level to model the quality of functional test sequences. This metric is based on the transition counts, observability vectors, the weight vectors and the threshold value for registers. By adopting the deviation metric, timing-consuming fault simulation at gate-level can be avoided for the grading of functional test sequences. Experiments on the Biquad filter and the Scheduler module of the IVM design show that the deviations obtained at RT-level correlate well with the stuck-at, transition and bridging fault coverage at the gate level. Moreover, the functional test sequences set reordered using deviations provides a steeper cumulative stuck-at and transition fault coverage as well as $B C E+$ metric, and there is an order of magnitude reduction in CPU time compared to gate-level methods.

\section{REFERENCES}

[1] M. L. Bushnell and V. D. Agrawal, Essentials of electronic testing for digital, memory, and mixed-signal VLSI circuits, Boston; Kluwer Academic Publishers, 2000.

[2] P. C. Maxwell, I. Hartanto and L. Bentz, "Comparing functional and structural tests," in Proc. Int. Test Conf., pp. 400-407, 2000.

[3] A. K. Vij, "Good scan= good quality level? well, it depends...," in Proc. Int. Test Conf., pp. 1195, 2002.

[4] J. Gatej et al., "Evaluating ATE features in terms of test escape rates and other cost of test culprits," in Proc. Int. Test Conf., pp. 1040-1049, 2002.

[5] J. Rearick and R. Rodgers, "Calibrating clock stretch during AC scan testing," in Proc. Int. Test Conf., pp. 266-273, 2005.

[6] P. A. Thaker, V. D. Agrawal, and M. E. Zaghloul, "Register-transfer level fault modeling and test evaluation techniques for VLSI circuits," in Proc. Int. Test Conf., pp. 940-949, 2000.

[7] F. Corno, M. S. Reorda and G. Squillero, "RT-level ITC'99 benchmarks and first ATPG result," in IEEE Design \& Test of Computers, pp. 44-53, 2000.

[8] M. B. Santos, F. M. Goncalves, I. C. Teixeira, and J. P. Teixeira, "RTLbased functional test generation for high defects coverage in digital systems," in Journal of Electronic Testing: Theory and Applications, vol. 17, pp. 311-319, 2001.

[9] W. Mao and R. K. Gulati, "Improving gate level fault coverage by RTL fault grading," in Proc. Int. Test Conf., pp. 150-159, 1996.

[10] S. Park et al.,"A functional coverage metric for estimating the gate-level fault foverage of functional tests," in Proc. Int. Test Conf., 2006.

[11] I. Pomeranz, P. K. Parvathala, S. Patil, "Estimating the fault coverage of functional test sequences without fault simulation," in Proc. Asian Test Symposium, pp. 25-32, 2007.

[12] T. E. Marchok et al., "A complexity analysis of sequential ATPG," IEEE Trans. CAD, vol. 15, pp. 1409-1423, Nov. 1996

[13] I. Pomeranz and S. M. Reddy, "LOCSTEP: a logic-simulation-based test generation procedure," IEEE Trans. CAD, vol. 16, pp. 544-554, May 1997.

[14] Z. Wang and K. Chakrabarty, "Test-quality/cost optimization using output-deviation-based reordering of test patterns," IEEE Trans. CAD, vol. 27, pp. 352-365, 2008.

[15] Z. Wang, H. Fang, K. Chakrabarty and M. Bienek, "Deviation-based LFSR reseeding for test-data compression," IEEE Trans. CAD, vol. 29, pp. 259-271, Februrary 2009.

[16] H. Fang, K. Chakrabarty and H. Fujiwara, "RTL DFT techniques to enhance defect coverage for functional test sequences," Journal of Electronic Testing: Theory and Applications, vol. 26, pp. 151-164, April 2010.

[17] Z. Navabi, VHDL: Analysis and modeling of digital systems, New York; McGraw-Hill Companies, 1997.

[18] Biquad Infinite Impulse Response Filter, http: //opencores.com/project,biquad/.

[19] N. J. Wang et al., "Characterizing the effect of transient faults on a high-performance processor pipeline," in Proc. Int. Conf. Dep. Sys. and Net., pp. 61-70, 2004.

[20] N. J. Wang and S. J. Patel, "Restore: symptom based soft error detection in microprocessors," in Proc. Int. Conf. Dep. Sys. and Net., pp. 30-39, 2005.

[21] M. Maniatakos et al., "Design and evaluation of a timestamp-based concurrent error detection method (CED) in a modern microprocessor controller," in Proc. Int. Symp. DFT in VLSI Systems, 2008.

[22] N. Karimi et al., "On the correlation between controller faults and instruction-level errors in modern microprocessors," in Proc. Int. Test Conf., 2008.

[23] B. J. Chalmers, Understanding statistics; CRC Press, 1987. 
[24] Documentation for the Parwan processor, http: //mesdat.ucsd.edu/ lichen/260c/parwan/.

[25] B. Benware et al., "Impact of multiple-detect test patterns on product quality," in Proc. International Test Conference, pp. 1031-1040, 2003.

[26] H. Tang et al., "Defect aware test patterns," in Proc. Design, Automation, and Test in Europe Conf., pp. 450-455, 2005.

[27] H. Fang et al., "RT-level deviation-based grading of functional test sequences," in Proc. VLSI Test Symp., pp. 264-269, 2009.

[28] N. Yazdani and Z. Ozsoyoglu, "Sequence matching of images," in Proc. International Conference on Scientific and Statistical Database Systems, pp. 53-62, 1996.

[29] M. Lu and H. Lin, "Parallel algorithms for the longest common subsequence problem," in IEEE Trans. on Parallel and Distributed Systems, vol. 5, pp. 835-848, 1994.

[30] G. Kalton, "Introduction to survey sampling," SAGE University Paper series on Quantitative Applications in the Social Sciences, series no. 07-035, Beverly Hills; SAGE Publications, 1983. 